\title{
LA RESPONSABILIDAD SOCIAL Y EL TURISMO SOSTENIBLE; RESPONSABLE
}

\author{
PROF. DRA. ELENA DEL PILAR RAMALLO MIÑÁN ${ }^{1}$ \\ DIRECTORA DE LA CÁTEDRA INTERNACIONAL DE RSC DE LA \\ EUROREGIÓN GALICIA-NORTE DE PORTUGAL \\ -FUNDACIÓN CEER-
}

\section{Resumen}

La literatura académica sobre la Responsabilidad Social (en adelante RS) dígase RSC (Corporativa) o RSE (Empresarial) en las últimas décadas se ha incrementado, aunque son todavía escasos los trabajos y mucho más si buscamos sectorialmente las investigaciones.

Al realizar una revisión sobre lo más destacado que se ha publicado, observamos que en el sector turístico no abundan los estudios y análisis de caso sobre la gestión de la RS en ésta sobresaliente industria para nuestro país.

El objetivo principal de este artículo, que realiza una breve retrospectiva sobre algunos estudios y posiciones de la RS, es plantear una línea de publicación sobre ambas temáticas, la RS y el turismo. De este modo, esta revista que con este número comienza su andadura, apostaría por la investigación en el turismo del futuro; el turismo sostenible.

\section{Abstract}

The academic literature on Social Responsibility (hereinafter RS) says CSR (Corporate) or CSR (Business) in the last decades has increased, although there are still few jobs and much more if we seek sectorial research.

When making a review on the most outstanding that has been published we observed that in the tourism sector there are many studies and case studies on the management of SR in this outstanding industry for our country.

The main objective of this article, which makes a brief retrospective about some studies and positions of the RS, is to propose a publication line on both themes, RS and tourism. In this way, this magazine, which with this issue begins its journey, would bet on research into future tourism, sustainable tourism.

Palabras clave: Turismo sostenible, turismo responsable, industria turística, responsabilidad social.

\footnotetext{
1 elenaramallom@correo.udc.es
} 
Key words: Sustainable tourism, responsible tourism, tourist industry, social responsibility.

\section{Breve revisión}

En este tema de creciente interés en la sociedad, en la Responsabilidad Social, como en todo, hay distintos posicionamientos. Los que aquí se revisarán son los que plantean la RS como estratégica, concebida como parte esencial de la empresa, por considerar esta autora que son los que mayor interés despiertan en el ámbito empresarial en general y en el sector del turismo en particular.

Para esta aproximación a las distintas teorías y autores sobre este ámbito estratégico, resulta imprescindible la mención al artículo de Daniela Toro (2006) que realiza una magnífica revisión a las más destacadas.

De los que se mencionan en el artículo de Toro (2006) referenciaré a Burke y Logsdon (1996) para exponer el concepto de la RSC como estrategia metodológica en la empresa (Burke y Logsdon, 1996) y a Husted y Allen (2000 y 2001), pero también recurriré a otros al margen de este artículo recopilatorio que he considerado interesante mencionar, como son Carroll (1991), Quazi y O'brien (2000) y Garriga y Melé (2004).

Burke y Logsdon (1996). Estos autores plantean el modelo de gestión estratégica de los stakeholders y la inclusión de las demandas sociales como aspectos estratégicos con consecuencias positivas para el desempeño financiero de la empresa. Exponen que para que la RSC sea estratégica debe estar relacionada con la misión de la empresa y, por tanto, con su plan estratégico, sin desatender los intereses de los stakeholders de manera global. De este modo la RSC es estratégica cuando aporta beneficios con el ejercicio propio empresarial, en especial cuando apoya actividades centrales y contribuye a la efectividad de la empresa en alcanzar su misión.

Burke y Logsdon (1996) identificaron cinco dimensiones de la RSC que reorienta los intereses económicos de la empresa y a los de sus stakeholders, desde la perspectiva de que las actividades caracterizadas por su centralidad, específicidad, proactividad, voluntariedad y visibilidad son las idóneas para promover valor a la empresa.

Husted y Allen (2000 and 2001) exponen que la RS y la estrategia del negocio han sido percibidas tradicionalmente de manera separada en la empresa, lo que no es acertado ya que el diseño de una estrategia de RSC incorporada en la estrategia de negocio de la empresa diferencia a las empresas y les permite construir ventajas competitivas.

Consideraron que la centralidad, la aproximación entre la política o programa de RSC y la misión y objetivos de la empresa son claves para obtener beneficios competitivos.

No obstante esta variable es un tema debatido en numerosas definiciones de estrategia que no han logrado grandes apoyos a la tesis expuesta.

Husted y Allen (2000) continúan apoyando el concepto de estrategia social que contribuye a la creación de valor para la empresa.

Husted y Allen (2000) plantean, también, que una empresa genera más valor en aquellos proyectos estratégicamente principales. Cuando la empresa no desarrolla esta 
perspectiva estratégica y el proyecto no es principal la empresa no tiene la pujanza ni el conocimiento para lograr el éxito estratégico, por lo que la elección de estos proyectos debe valorarse con suma atención.

Estos autores defienden, asimismo, la vinculación de la RS y la I+D de aquellas empresas que desarrollan estrategias sociales con su oferta de productos para sus clientes, consiguiendo, de este modo, ventajas competitivas y valor añadido.

En la investigación de Husted y Allen (2001) se aborda el modelo de estrategia social corporativa desde el punto de vista de la relación de la RSC y el ámbito de negocio de la empresa. Para ello desarrollaron una estrategia social basándose en cuatro elementos: 1) la estructura de la industria, 2) los recursos de la empresa, 3) la ideología y valores corporativos y 4) los stakeholders.

Husted y Allen (2001) comienzan por definir qué entienden por estrategia social corporativa, compuesta por los objetivos económicos (estrategia de negocio corporativa) y no-económicos (estrategia social corporativa) de la empresa y diseñando tres estrategias sociales genéricas. Posteriormente analizan la relación entre cada uno de ellos en la estrategia social y con las estrategias genéricas, así como el impacto que esto genera en la creación de ventaja competitiva para la empresa. Entendiendo la estrategia como planes, inversiones y acciones realizadas para alcanzar ventajas competitivas sostenibles que llevarían a un óptimo desempeño financiero y social. Toro (2006)

La estrategia de negocio se refiere a los aspectos económicos y a la estrategia social. En base a ello, definen la estrategia social como el posicionamiento de la empresa con respecto a los temas sociales para poder alcanzar objetivos sociales a largo plazo y crear ventajas competitivas. Toro (2006)

Para Carroll (1991) las acciones de RS en la empresa se concentran en cuatro bloques; las responsabilidadeseconómicas, que cumplen el triple propósito de satisfacer al cliente con productos de buena calidad, generar buenos trabajos a los empleados y mejorar la rentabilidad para el accionista; las legales que se enfocan en el cumplimiento de las leyes y las regulaciones gubernamentales propias de la actividad empresarial, y las éticas que son guiadas por la moral de la empresa e implican una actuación correcta, justa y equitativa con los diferentes grupos de interés. Y todas ellas, para cumplir su función y obtener los objetivos establecidos, deben formar parte de la estrategia de la empresa.

Quazi \& O'brien (2000) afirman que la RS en la empresa, desde una perspectiva moderna, simboliza una permuta en las actuaciones empresariales que afecta transversalmente a todos los ámbitos de la organización. De este modo, la RSE es la estrategia adecuada para situar a la empresa en un modelo de gestión organizacional responsable que reduce los impactos negativos generados por la actividad empresarial a la vez que genera valor añadido, tanto para la empresa como a sus grupos de interés, stakeholders, $\mathrm{y}$ a la sociedad en general.

Los planteamientos de Garriga \& Melé (2004) partían de la clasificación de las actividades y enfoques de la RSE en cuatro grupos de teorías relacionadas con los beneficios; (Teorías Instrumentales), la actuación política (Teorías Políticas), las demandas sociales (Teorías Integradoras) y los valores éticos (Teorías Éticas). En el primer grupo se concentrarían los autores que entienden la RS desde un enfoque instrumental. El desarrollo de un comportamiento responsable y ético, siempre y cuando aporte beneficios y ventajas competitivas. En caso de que esto no sea así se abandonará 
esta estrategia.El segundo enfoque reúne a aquellas teorías que se centran en el poder de las corporaciones en la sociedad y el uso responsable de este poder en la política. El tercer enfoque lo conforman aquellas teorías integrativas según las que la empresa se centra en la satisfacción de las demandas sociales de sus stakeholders. El último enfoque agrupa las teorías que abordan la responsabilidad ética de las empresas frente a la sociedad.

De los cuatro enfoques mencionados interesa resaltar el que expone las teorías instrumentales, pues ya dentro de este grupo se incluyen estudios que reconocen el carácter estratégico de la responsabilidad social en la medida en que sea beneficioso para el negocio y contribuya a alcanzar objetivos económicos y ganancias.

\section{El turismo responsable es turismo sostenible}

La otra cuestión que aborda este artículo es el turismo, concretamente el turismo sostenible. El turismo es una industria destacada en el mundo y principal en España. El turismo promueve la creación de empleo, incrementa los ingresos, permite el desarrollo de las zonas, desarrolla los intercambios culturales, la apertura hacia otros modos de vida y culturales....Pero la industria turística genera una serie de impactos negativos que se clasifican en tres grandes grupos; los económicos, los socioculturales y los ambientales. En cuanto a estos últimos, Lluill,A (2001) en su tesis doctoral, Contabilidad medioambiental y desarrollo sostenible en el sector turístico, realiza un análisis sobre los impactos ambientales, cuya clasificación debe realizarse desde el punto de vista del consumo de inputs y de la generación de outputs. Los impactos producidos como consecuencia del primero se pueden dividir en tres niveles; los derivados de la fase de construcción e implantación del equipamiento turístico y las infraestructuras; los producidos en su estado de funcionamiento operativo; y los generados por las actividades que realizan los propios turistas.

Por lo tanto, las consecuencias negativas de esta actividad provocan la degradación de los recursos naturales y el incremento en el consumo agua, energía, residuos, necesidad de tratamiento de fecales, alteración de los ecosistemas, introducción de especies exóticas de animales y flora no autóctona, aumento de incendios..., así como la alteración urbanística de los lugares y zonas de especial interés natural y patrimonial, por el aumento, en algunos casos desaforado, de flujos de población hacia estas zonas, que acaban alterándolas e incluso a veces destruyéndolas.

Estos efectos negativos, las nuevas tendencias de viajes y la concienciación, cada vez más numerosa de un turista responsable, provocan que el turismo se encamine hacia el turismo sostenible.

El turismo sostenible representa la gestión sensata de los recursos naturales, económicos y culturales de una región. La OMT, ante el turismo sostenible, afirma que éste es "un modelo de desarrollo económico concebido para mejorar la calidad de vida de la comunidad receptora, para facilitar al visitante una experiencia de alta calidad y mantener la calidad del medio ambiente, del que tanto la comunidad anfitriona como los visitantes dependen” (OMT,1998:260).

Por lo tanto, la sostenibilidad en el turismo no puede plantearse como una forma de diferenciación empresarial ni, por supuesto, como un turismo de naturaleza y ya.

La sustentabilidad debe acompañar al desarrollo de la industria turística y concebirse como una de sus condiciones básicas para transformar a la actividad en una oportunidad de cambio que genere riqueza cultural y social, una mejor distribución del 
ingreso y un aprovechamiento de los recursos naturales y culturales, permitiendo su subsistencia en el tiempo.

Según la Organización Mundial del Turismo -OMT-, los principios que definen el turismo sostenible son:

- Se conservan los recursos naturales y culturales para su uso continuado en el futuro, al tiempo que reportan beneficios.

- El desarrollo turístico se planifica y gestiona de forma que no cause serios problemas ambientales o socioculturales.

- La calidad ambiental se mantiene y mejora.

- Se procura mantener un elevado nivel de satisfacción a los visitantes y el destino retiene su prestigio y potencial comercial.

- Los beneficios del turismo se reparten ampliamente entre toda la sociedad.

Continuando la línea argumentativa del turismo sostenible y con la idea de unirlo con el concepto de RS, referenciamos la publicación del Libro verde en el año 2001 por parte de la Comisión Europea, en el cual se trata de iniciar un debate con los objetivos de fomentar la Responsabilidad Social Empresarial de las empresas en los ámbitos europeo e internacional a la vez que se trata de indagar sobre cómo aprovechar las experiencias existentes, fomentar el desarrollo de prácticas innovadoras, aumentar la transparencia e incrementar la fiabilidad de la evaluación y la validación para todas aquellas empresas que la quieran implantar o mejorar. A lo largo de todo el Libro verde se dan pautas sobre aspectos relacionados con la RS, tanto con la dimensión interna como externa de la empresa. Este libro consta de dos partes (cuyos temas serán tratados a lo largo del artículo) subdivididas a su vez en capítulos. Se aborda la integración de la gestión de la responsabilidad social en la estrategia organizacional, la elaboración de informes de sostenibilidad, el etiquetado de productos y servicios, la calidad en el trabajo y la inversión socialmente responsable.

La primera parte del Libro se centra en el ámbito interno de la empresa y trata temas tan relevantes como la gestión de recursos humanos, la salud y la seguridad en el ámbito del trabajo, la adaptación al cambio o la gestión del impacto medioambiental y de los recursos materiales. En la segunda parte se aborda el ámbito externo, tratando temas como el de las comunidades locales, el de los socios comerciales, proveedores y consumidores, además de los problemas ecológicos mundiales. En este sentido, en la Cumbre Mundial sobre el Desarrollo Sostenible, celebrada en Johannesburgo, África, en 2002, la OMT dice que el "desarrollo sostenible consiste en lograr el equilibrio entre los objetivos sociales, económicos y ambientales” y la Resolución del Consejo de la Unión Europea del 6 de Febrero del 2003, expone que la estrategia de RS de las empresas debe basarse en los siguientes puntos: el reconocimiento de la naturaleza voluntaria de la responsabilidad social de la empresa, la necesidad de que las prácticas de responsabilidad social sean dignas de crédito y transparentes, la focalización en actividades en las que la participación de la comunidad suponga una ventaja y el planteamiento equilibrado y amplio que incluya aspectos económicos, sociales y medioambientales. Al mismo tiempo se compaginan los intereses de los consumidores, la atención a las necesidades específicas y a las características de la pequeña y mediana empresa y el apoyo a los instrumentos existentes acordados internacionalmente y su compatibilidad con ellos.

Al hilo de estas resoluciones y manifestaciones institucionales, cabe recordar asimismo que el Código Ético Mundial para el Turismo, que consta de 10 principios, 
aborda los aspectos económicos, sociales, culturales y ambientales de los viajes y el turismo y, en su apartado tercero, referencia el turismo como un factor de desarrollo sostenible.

Desde este punto de vista, Tepelus (2008) considera la RSE como un marco conceptual mentor para avanzar en la temática sobre la sostenibilidad del turismo determina la responsabilidad específica para el sector.

\section{Conclusiones}

Por lo tanto, para que la RS pueda ser entendida como una estrategia social capaz de generar ventajas competitivas que tengan un impacto positivo en la rentabilidad de la empresa, pero que éstas obtendrán resultados no inmediatos, debe plantearse como una inversión a largo plazo.

La estrategia debe ser diseñada como eje central de la empresa concibiendo los objetivos sociales y los financieros al mismo nivel. A la vez que deben conectarse las sinergias entre la I+D y el diseño de programas sociales incluyendo las demandas de los stakeholders.

Asimismo, resulta necesario mencionar que en una empresa cuya actividad económica es reconocida por el cumplimiento de estándares de calidad y por su aportación social y ambiental, el posicionamiento competitivo y la proyección pública serán valores añadidos al desarrollo de su actividad, que la diferencia y posiciona de manera preferente en el mercado.

La industria turística debe ver en la RS una oportunidad empresarial de presente y de futuro y deben promover acciones más allá del cumplimiento de las leyes y las normas. Debe de incorporarla a su estrategia global y expenderla en todas sus áreas.

\section{Bibliografía}

Aparicio, J. y Valdés, B. (2009) Sobre el concepto de responsabilidad social de las empresas. Un análisis europeo comparado. Cuadernos de relaciones laborales 27(1), 53-75.

Aragón, J. y Rocha, F. (2009). Los actores de la responsabilidad social empresarial. Cuadernos de relaciones laborales, 27(1), 147-167.

Borjas, C. (2007) Responsabilidad social empresarial y desempeño financiero: un enfoque estratégico. Revista de ciencias empresariales y economía, 6, 137-14.

Boza, J. y Pérez, D. (2009) Estudio de la responsabilidad social de las empresas. Aplicaciones a las empresas de Gran Canaria. EAWP: Documentos de trabajo en análisis económico, 8(4), 29-38.

Castellano, M., Martín, I. y Caro, F. J. (2007) Propuesta de una escala de medición de la responsabilidad social corporativa (RSC) en la actividad turística. Conocimiento, innovación y emprendedores: camino al futuro, 2621-2632.

De la Cuesta, M. (2004) El por qué de la responsabilidad social corporativa. Información comercial española (ICE), 2813, 45-58.

De la Cuesta, M. y Valor, C. (2007) Condiciones para la eficacia de los códigos internos de responsabilidad social de la empresa, Ekonomiaz, 65, 128-149.

Fernández, R. y Martínez, A. (2008) Naturaleza estratégica de la responsabilidad social empresarial. Revista de globalización, competitividad y gobernabilidad, 2(2), 45-57. 
García, V. D. (2004) La responsabilidad social de la empresa: una definición desde la ética empresarial. Revista valenciana de economía y hacienda, 12, 77-98.

González, L. (2004) La responsabilidad social de la empresa. Revista del Ministerio de Trabajo e Inmigración, 50, 13 - 20.

Morales, H. I. (2005) Responsabilidad social de las empresas. Revista latinoamericana de derecho social, 1, $149-179$.

Organización Mundial del Turismo-OMT (2004): “Conceptos y Definiciones - Desarrollo Sostenible del Turismo” - Definición conceptual (agosto de 2004), http://www.worldtourism.org/sustainable/esp/concepts.htm

Redondo, H. (2005) Cómo integrar la responsabilidad social en la cultura empresarial. Revista asturiana de economía, 34, 31-41.

Rodríguez, O., Fernández, J. y Martín, J. I. (2007) Percepción directiva de las relaciones entre responsabilidad social y ética empresarial. Información comercial española (ICE), 2927, 4356.

Tepelus,C.M.(2008). Destination Unknown? The Emergence of Corporate Social Responsibility for Sustainable Development of Tourism. Tesis Doctoral. Lund University. Sweden.

Toro, D (2006) El enfoque estratégico de la responsabilidad social corporativa: revisión de la literatura académica. Intangible Capital № 14 - (Vol. 2) 338-358

Valor, C. y De la Cuesta, M. (2003) Responsabilidad social de la empresa, medición y desarrollo en España. Información comercial española (ICE), 2755, 7-20.

Valor, C., Kreisler, I. y De la Cuesta, M. (2003) Integración de la responsabilidad social de la empresa en el cuerpo legislativo español. Economistas, 21(98), 219-224. 